\title{
La hermenéutica en el desarrollo de la investigación educativa en el siglo XXI
}

Claudia Barrero Espinosa* Liliana Bohórquez Agudelo Martha Patricia Mejia Pachón

Resumen. El presente texto tiene como propósito fundamental hacer una interpretación relacionada con el estudio de la realidad de la investigación educativa, basada en las diferentes miradas que plantean algunos autores tomados como referentes para comprender e interpretar las acciones de los agentes educativos frente a los paradigmas o enfoques que tradicionalmente se han mantenido implícitos en el campo de la metodología de la investigación. Se apunta a renovar las creencias como reto que tiene la universidad colombiana para el siglo XXI, puesto que la figura del docente actual va más allá de su labor en el aula, superando las barreras epistemológicas, sociales y humana. Esto le permite construir conocimiento, generando un cambio en sus prácticas y empleando diferentes estrategias investigativas que fortalezcan las ciencias sociales.

Palabras clave. Investigación educativa, paradigmas, interpretación, teoría, práctica, comunicación.

* Profesoras de la Universidad de San Buenaventura. Candidatas a Magister en Educación de la Universidad de Santo Tomás. barreroclauida@hotmail.com 


\begin{abstract}
The present text has as fundamental intention do an interpretation related to the study of the reality of the educational investigation, based on the different looks that some authors taken as models raise to understand and to interpret the actions of the educational agents opposite to the paradigms or approaches that traditionally have been kept implicit in the field of the methodology of the investigation, pointing to renew the beliefs as challenge that has the Colombian university for the 21st century, since the figure of the current teacher goes beyond his labor in the classroom, overcoming the barriers epistemological, social and human that allow him to construct knowledge generating a change in his practices and using different strategies.
\end{abstract}

Key Words. Educational research paradigms, interpretation, theory, practice, communication.

\title{
Introducción
}

El planteamiento interpretativo de la teoría y la práctica educativa, ideas desarrolladas por los autores Carr Wilfred y Kemmis Stephen crean la necesidad de reflexionar críticamente sobre la práctica pedagógica como actividad investigativa desarrollada sin interrupción por muchos años; es el origen de estas reflexiones desde la interpretación de los diferentes paradigmas que en función de la posición filosófica fundamentan la supremacía o valor primordial de algunos de los paradigmas. La calidad de este tema, no sólo es de orden teórico, sino que también tiene una trascendencia práctica, en una situación como la actual, en la que cada vez un mayor número de docentes se interesa por participar en las investigaciones, ya que aumenta la comprensión del papel de éstas como elemento fundamental en el perfeccionamiento del proceso docente. 


\section{A. Paradigmas de los enfoques positivista $\epsilon$ interpretativo}

Algunas de las posiciones teóricas sobre la concepción de los paradigmas como la postura de Thomas Kuhn son las realizaciones científicas que durante cierto tiempo proporcionan modelos de problemas y soluciones a una comunidad científica (1971). Paradigma, según Ritzer es "una imagen fundamental del objeto de estudio dentro de una disciplina. Sirve para definir lo que debe estudiarse, qué cuestiones deben preguntarse, cómo debe preguntarse y qué reglas deben seguirse al interpretar las respuestas obtenidas. El paradigma es la unidad más amplia del consenso dentro de una ciencia y sirve para diferenciar una comunidad científica con otra. Define e interrelaciona los casos ejemplares, teorías, métodos e instrumentos que existen dentro de ella" (1975).

Algunos autores prefieren sustituir el término de paradigma por el de programa de investigación, concepto introducido por Lakatos (1983). El concepto de programa de investigación se refiere a una descripción de los distintos géneros de investigación según las decisiones del investigador Guillermo Orozco, quien señala la existencia de cuatro paradigmas de producción de conocimientos vigentes en la actualidad para las ciencias sociales. Las principales diferencias entre ellos según este autor se expresan en su intencionalidad y en la manera de producir conocimientos: el positivista marcado por un interés en la verificación y en la comprobación. El realista que se centra en las causas de los fenómenos. El hermenéutico que radica en la interpretación del objeto de estudio y el interaccionista que busca la conexión de los elementos que influyen en el objeto de estudio. (1997)

La investigación educativa ha fallado en sus intentos de pronosticar la evolución de la práctica educativa en la escuela, de obtener resultados que permitan trazar con suficiente antelación políticas encaminadas a perfeccionar la educación y no ha podido 
en muchos campos ofrecer una explicación acertada sobre el desarrollo de fenómenos pedagógicos. Hay que agregar además el absoluto divorcio entre investigadores y docentes que ha existido en un período en fase de superación; pero prolongado en la historia de la investigación educativa. Esta independencia ha tenido consecuencias, la no atención por parte de los investigadores a los problemas que son considerados fundamentales por los docentes $\mathrm{y}$, como consecuencia, la falta de compromiso de investigadores $\mathrm{y}$ docentes con la aplicación de los resultados de las investigaciones en la práctica.

El paradigma positivista desconoce la cientificidad de otras formas de enfocar el estudio de la realidad educativa, es entonces cuando adopta de antemano una supremacía a favor de este paradigma. Este paradigma ha influido en las investigaciones educativas por mucho tiempo. No hay distinción entre los fenómenos de la naturaleza y los sociales, considerando la realidad educativa como única y estable, sin valorar la complejidad de su objeto de estudio que la diferencia esencialmente de las ciencias naturales. Reconoce la educación y su práctica como fenómenos que deben ser estudiados objetivamente, a través de una comprensión instrumental y técnica al estilo positivista.

Las investigaciones basadas en este paradigma consideran la vía hipotética deductiva como la más efectiva para todas las investigaciones. El trabajo científico tiene como finalidad esencial el establecimiento de leyes por las que se rigen los fenómenos. Según esta concepción dichas leyes pueden ser descubiertas y explicadas imparcialmente y admiten revelar, predecir y controlar el fenómeno objeto de estudio. En el caso de las investigaciones educativas, trata de explicar y predecir las conductas de los sujetos implicados en los fenómenos educativos, también reconoce el conocimiento como válido sólo cuando es objetivo, es decir independiente de quien lo descubre, considerando, por tanto, la actividad científica como desinteresada y diferencia entre papel del investigador y las 
funciones del docente. El investigador es considerado un agente externo que se desempeña como experto, reconocido como sujeto y determina el objeto de estudio, por lo cual genera la idea investigativa desde fuera, sin tomar en consideración las expectativas de las personas implicadas y que son consideradas como objetos de investigación.

Por lo tanto, aspiran a ampliar el conocimiento teórico, asignándole a la teoría el papel de guiar la práctica. La determinación de los principios y leyes del proceso educativo permitirá aumentar la eficiencia de éste sobre la base de la teoría científica. En este paradigma los diseños de investigación se caracterizan por ser formales y estáticos a partir del manejo del conjunto de variables que pretende estudiar. Se utilizan predominantemente técnicas cuantitativas con la intención de alcanzar la mayor precisión y exactitud. Las investigaciones culminan con un informe final en el que se expone a la comunidad científica los resultados obtenidos, en un lenguaje alejado de aquéllos que deberán aplicar en las aulas dichos resultados. En este informe se recogen indicaciones para los educadores con el fin de que a partir de estos conocimientos científicos transformen la práctica educativa con resultados positivos.

El paradigma positivista diferencia claramente tres momentos importantes: la producción del conocimiento, su instrumentación y su aplicación en la práctica. El paradigma positivista es reduccionista; al hacer equivalentes los fenómenos naturales y los sociales, pierde de vista la riqueza y complejidad de estos últimos, se desconoce la activa participación del hombre en estos procesos y se sacrifica a la objetividad científica el análisis de la amplia escala de interacciones que siempre está presente en los fenómenos sociales, sin tener en cuenta la presencia de variables incontrolables, la conformación en sistema de estos fenómenos, la complejidad de la psiquis humana y de la conformación de los grupos de estudiantes. En estas condiciones no quedan claro cuántas observaciones son necesarias para verificar la hipótesis propuesta. Se destaca 
además como una limitación que los resultados obtenidos a través de investigaciones realizadas siguiendo este paradigma, se han caracterizado por ofrecer cuerpos de conocimientos teóricos que se alejan de la práctica educativa y no contribuyen a la solución de los problemas apremiantes. Los docentes no se sienten partícipes en su concepción y desarrollo.

Al comparar los resultados de su aplicación en las investigaciones educativas, con los obtenidos en las ciencias naturales se argumenta en estos últimos su repetición por diferentes vías que conducen al conocimiento. En las investigaciones educativas es imposible esta concepción del experimento, por cuanto se trabaja con seres humanos a los cuales, por razones de ética científica, no se les puede permitir afectaciones en el desarrollo. Al considerar el paradigma positivista la actividad científica como desinteresada y neutra, le niega el componente ideológico que posee en la esfera social -en general-y educacional en particular. De igual manera, señala como una limitante que su tendencia a establecer generalizaciones le lleva a olvidar la individualidad, que tanta importancia tiene para la labor educativa, ya que la labor del maestro no está dirigida solamente a lograr los resultados más efectivos con el grupo en general, sino a obtener de cada uno de sus estudiantes los mayores progresos posibles.

En este sentido el paradigma interpretativo-hermenéutico parte de reconocer la diferencia existente entre los fenómenos sociales y naturales, buscando la mayor complejidad y el carácter inacabado de los primeros, que están siempre condicionados por la participación del hombre. Abarca un conjunto de corrientes humanísticointerpretativas, cuyo interés fundamental va dirigido al significado de las acciones humanas y de la vida social. Concibe la educación como proceso social, como experiencia viva para los involucrados en los procesos y para las instituciones educativas, enfatiza que transformando la conciencia de los docentes, éstos transformarán su práctica educativa. 
El paradigma interpretativo intenta hacer una negación de las nociones científicas de explicación, predicción y control del paradigma positivista, las cambia por las nociones de comprensión, significado y acción. Sus propósitos esenciales están dirigidos a la comprensión de la conducta humana a través del descubrimiento de los significados sociales. Aspira a penetrar en el mundo personal de los hombres, cómo interpretar las situaciones, qué significan para ellos, qué intenciones, creencias y motivaciones los guían. Su objeto de estudio fundamental son las interacciones del mundo social, enfatizando el análisis de la dimensión subjetiva de la realidad social, a la cual comprende como un conjunto de realidades variadas. Este paradigma considera la realidad educativa como subjetiva, persigue la comprensión de las acciones de los agentes del proceso educativo. La práctica educativa puede ser transformada si se modifica la manera de comprenderla. Las investigaciones realizadas según este paradigma se centran en la descripción y comprensión de lo particular, lo singular de los fenómenos, más que en lo generalizable. No aspira a encontrar regularidades subyacentes en los fenómenos, ni el establecimiento de generalizaciones o leyes. El investigador describe las acciones contextualizadas. No busca nexos causales, sino comprende las razones de los individuos para percibir la realidad de una forma dada.

En contraste con la tendencia positivista a estudiar los fenómenos según lo observable y a la aplicación de técnicas de procesamiento cuantitativo de la información, este paradigma dirige su atención a aquellos aspectos no observables ni susceptibles de cuantificación, como opiniones, propósitos, motivaciones, comentarios, significados. Interesa lo particular y lo contextual, los relatos vividos. Pretende desarrollar un conocimiento representativo y comprende la realidad como dinámica y diversa.

Este paradigma niega el carácter de la actividad científica, reconociendo la importancia que tiene la posición social e ideológica 
de las personas implicadas en la investigación. Los hechos se interpretan partiendo de los deseos, intereses y expectativas de los sujetos. Toma como metodología fundamental la vía inductivadeductiva para el desarrollo de la investigación y reconoce las teorías científicas no como universales, sino como dependientes del contexto social e histórico en que ocurren los fenómenos. Según este paradigma la validez del conocimiento viene dada como producto del consenso y se expresa a través del conocimiento tácito, los sentimientos y las intenciones. El criterio fundamental para determinar la validez de las investigaciones es su relevancia.

Dicho paradigma no tiene carácter formal y estático, sino que son diseños emergentes, propios del proceso del desarrollo de la investigación. Desde el punto de vista metodológico este paradigma recurre a la observación, entrevista y otros métodos, sin esquemas rígidos, sin intentar convertir sus resultados en cuadros que no reflejen la complejidad de la realidad estudiada. Entre las limitaciones que se le señala el peligro de conducir el conservalismo, si no se tiene en cuenta la necesidad de transformar la realidad como razón de ser de la ciencia; para lo cual es necesario tomar en consideración la interrelación dialéctica entre lo particular y lo general.

Los seguidores del paradigma positivista señalan que se apartan del método científico que exige ir más allá de los problemas prácticos, experimentación controlada, aplicabilidad de los resultados a situaciones más amplias y colocar dichos resultados frente a un organismo existente de generalizaciones. Se planeta que lo que se realiza realmente es un trabajo social.

Bajo las directrices de este paradigma se han desarrollado investigaciones que han perdido de vista la necesidad de una preparación efectiva de la comunidad para la participación en la labor investigativa, se corre el riesgo de que se utilice en ésta, pero sin una intención seria de lograr algo que transforme la realidad educativa. 
Siguiendo este paradigma educativo se conciben las líneas de investigación centradas en el docente y en el alumno. En el alumno se dirige al proceso de transformación cognoscitiva considerando la perspectiva psicológica y sociológica. En el docente están dirigidas a los procesos cognitivos y su relación con los marcos o concepciones alternativas y la toma de decisiones relacionadas con las teorías implícitas. Se estudian las actividades del profesor en las fases proactiva, interactiva y postactiva. Las investigaciones más importantes se relacionan con las secuencias interactivas y la construcción de conocimientos. Analiza la enseñanza como dependiente de los que el alumno entiende. Utiliza como método el análisis del discurso y su forma a través de los enfoques lingüístico y sociolingüístico.

Algunas consideraciones metodológicas a los paradigmas fundamentales de la investigación educativa en la formación de los investigadores y docentes toman parte activa en las investigaciones que se desarrollan, adquiriendo cada día mayor significación. Hoy en día, un gran número de docentes presenta intereses dirigidos en este sentido. A partir de lo anteriormente planteado, es imprescindible que al investigador educativo se le brinde una panorámica lo más profunda posible acerca de los principales postulados que caracterizan a cada uno de los paradigmas.

\section{B. "La teoría y la práctica educativa" el eterno dilema de la investigación moderna y convencional}

El propósito fundamental es hacer una interpretación relacionada con la lectura: "Planteamiento Interpretativo de la Teoría y la Práctica Educativa", ideas desarrolladas por los autores Carr Wilfred y Kemmis Stephen, donde se pretende comprender cómo desde la interpretación, entendida como una operación que ha sido aplicada a documentos escritos, se permite el análisis y la comprensión de los acontecimientos socio-históricos y procesos educativos de una cultura, teniendo en cuenta que dentro de este fenómeno se 
pregunta por el sentido que tienen las cosas y cuál es el significado que se le da y no por lo que son las cosas, es decir, su esencia (problema que plantea la fenomenología). Sobre la base de las consideraciones anteriores se propone entonces aclarar desde una mirada personal cómo se concibe la fenomenología social; cómo este proceso se discute desde la hermenéutica, es decir, desde el subjetivismo que caracteriza al ser humano y sus relaciones estudiadas a través de las ciencias sociales y la incidencia de la teoría y la práctica educativa como elemento de reflexión que surge desde la pedagogía y la sustenta.

En el marco de las observaciones anteriores la sociología de la educación, del funcionalismo y desde la orientación positivista, entendida como el análisis del comportamiento humano determinado por las leyes impersonales que funcionan lejos del control del individuo y sus interacciones; esta orientación separa la teoría de la práctica utilizando instrumentos para la experimentación, manipulación de variables, datos estadísticos y deductivos; su finalidad es conocer y explicar la realidad para predecirla y controlarla, analiza problemas surgidos de la teoría.

Con el tiempo, la sociología de la educación toma una nueva dirección donde se plantea que la sociedad no es un sistema independiente, sino que es una estructura interior significativa, formada y soportada por las actividades entendidas por sus miembros individuales. La sociedad sólo es real y objetiva en la medida en que sus miembros la definen como tal y se orientan ellos mismos hacia la realidad definida, es decir, que el lenguaje se convierte en el medio por el cual se realiza el acuerdo de los interlocutores y el consenso sobre las cosas, la comprensión y experiencia del mundo mantienen una fuerte relación y el lenguaje es la herramienta que revela la red de significados a partir de los cuales los miembros de la sociedad constituyen y reconstituyen el orden social; esta interpretación se plantea desde la tesis que propone Gadamer con la teoría de la neo-hermenéutica. 
Esta es una forma que utiliza el campo de la educación a través de la indagación donde los maestros por medio de los pre-saberes de sus estudiantes conocen cómo éstos se relacionan con el saber, teniendo en cuenta el conocimiento y la aptitud como conceptos organizadores socialmente establecidos. La investigación sociológica entonces se aborda de manera holística, permitiendo la comprensión e interpretación de los problemas surgidos en relación con la educación y con el grupo social, se da un intercambio dinámico entre la teoría y la práctica utilizando instrumentos como la observación participante y la entrevista, entre otros; analiza los datos en varias etapas: reducción, categorización, representación, validación e interpretación, ciclo que permite pasar la sociología de la educación del mero funcionalismo a la fenomenología (primacía de la experiencia subjetiva).

Al mismo tiempo los autores hacen alusión al planteamiento interpretativo de las ciencias sociales, el cual emerge como reacción al intento de desarrollar una ciencia natural de los fenómenos sociales. Se entiende el sujeto como actor que da cuenta de las situaciones comprendiendo su sentido, dejando de lado la explicación científica por el enfoque interpretativo llamado hermenéutica, el cual inicia en el siglo XVII y toma fuerza en los inicios del siglo $\mathrm{XX}$, promovida por Dilthey como la metodología de las ciencias culturales o morales, desvirtuando un poco la postura positivista. Es allí donde a través de la experimentación fundamenta el estudio de los fenómenos humanos, por esa razón algunos autores reflexionaron acerca de la naturaleza del acto de comprender, por lo cual se demuestra que el investigador es capaz de mantenerse fuera de su propia historia para interpretar los significados de los otros. Esto quiere decir que la hermenéutica no se preocupa tanto por la intención del actor, sino que toma la acción como una vía para dilucidar el contexto social de significado más amplio en el que está inmerso. La noción de significado subjetivo según Weber se comprende desde la íntima relación que existe entre la acción humana y su conducta, refiriéndose a ésta como un movimiento 
físico aparente. En el orden de las ideas anteriores se puede afirmar que la escuela vista como micro sociedad, planteamiento que aduce Delia Lerner, es un espacio abierto al diálogo entre sus actores (estudiantes y maestros) y de la sociedad de la que forman parte, permite acciones pedagógicas liberadoras para formar estudiantes críticos y docentes que mediante su actividad como intelectuales transformativos generen cambios en la cultura innovadora, creativa, capaz de reproducir relaciones sociales equilibradas (humanas y racionales). El cambio educativo constituye un desafío para los docentes, dado su papel y la responsabilidad social que tienen frente a una comunidad; sin embargo, el rol que se les asigna corresponde cada vez más a los ejecutores de aprendizaje devaluando su trabajo intelectual y crítico. Por ello Giroux reivindica al docente como intelectual transformativo que combine la reflexión con la práctica educativa. La interacción de la política y la pedagogía significarían entonces servirse de formas para que los estudiantes sean tratados como sujetos críticos que problematizan el conocimiento recurriendo al diálogo crítico y afirmativo apoyando la perseverancia por un mundo mejor para todos.

Después de lo expuesto anteriormente se puede afirmar que la ciencia social interpretativa busca descubrir los significados de la acción, esto implica entonces que el ser humano sea consciente de sus intenciones al momento de actuar. Se puede decir que el carácter social de las acciones surge de las redes de significados otorgadas por sus antecedentes históricos y las acciones en orden al contexto en el presente son las que permiten comprender la realidad.

En este orden de ideas se puede entrar a decir que para la investigación de las ciencias sociales y su interpretación lo teórico y lo práctico deben estar al mismo nivel, ya que al dar la claridad de las acciones de los individuos crean posibilidades de un cambio práctico, puesto que reducen los problemas de comunicación entre aquéllos cuyas acciones demuestran lo que ocurre en una 
situación determinada, al revelar de qué modo los protagonistas de dicha situación extraen el sentido de lo que hacen.

Esta teoría social interpretativa logra influir en la práctica de manera que los practicantes comprendan la situación y puedan definir la realidad en la que interactúan. Otro elemento que proporciona dicha teoría es la posibilidad de tener en cuenta las creencias y aptitudes de los individuos inherentes a su manera de pensar actual y que ésta influya sobre su práctica y cambie la manera de comprenderla.

De acuerdo a la lectura hecha del módulo, se ha podido aclarar que la ciencia social interpretativa busca educar, generando compromisos frente a las acciones de los individuos, transformando las conciencias y diferenciando los modos interpretados de la acción; la ciencia interpretativa reconoce la influencia de la acción a través de la mediación en la reflexión crítica de los agentes individuales.

La teoría y la práctica implican acciones que generen intercambios bidireccionales; la deliberación práctica está informada, no sólo por las ideas sino por las exigencias y experiencias que genera cada situación; estas situaciones prácticas ofrecen el fortalecimiento de habilidades que aportan al conocimiento del hacer del actor.

De otra parte, el propósito fundamental de la ciencia social interpretativa es la ilustración de la racionalidad entendida en un sentido crítico, moral y reflexivo. Se puede decir que la relación teoría-práctica es la praxis de la interpretación crítica que constituye el método de la comprensión que según Verstehen consiste en entender la realidad o el objeto a través del lenguaje que representa el comportamiento o relación del contexto por parte de las intenciones de los sujetos y sus intereses. Va de lo particular por la acción del individuo a lo general a través de la dialéctica y el lenguaje al interactuar con el otro. Esta relación horizontal teoría-práctica permite comprender la relación lógica desde la 
dialéctica planteada por Gadamer, hasta el análisis-síntesis crítico y deducción-inducción.

En este sentido, el paradigma hermenéutico perfila un docente líder importante para la comunidad y que comprende la responsabilidad social que le compete. El desarrollo curricular que propende es integrador, flexible, define los roles de manera informal y se fundamenta en las acciones humanas. El fenómeno educativo es subjetivo, ya que depende de los conceptos que cada ser humano ha construido en la escuela o en la comunidad a la cual pertenece, le da importancia al contexto, es interactivo, vital, personal y cultural.

Para concluir, se puede afirmar que existen autores que plantean que el enfoque interpretativo de la relación teoría-práctica carece de solidez, ya que incorpora supuestos conservadores acerca de la relación entre el conflicto y el cambio social porque tiende a confundir su acción con el fenómeno que presenta la realidad por la misma subjetividad y sensibilidad del sujeto. El enfoque interpretativo siempre está predispuesto a favor de la idea de buscar que las personas encuentren una afinidad o consonancia con la realidad social presente.

En cuanto a la investigación educacional interpretativa, ésta resalta las interpretaciones subjetivas de los practicantes de la educación y sus realidades, poniendo en gran cuestionamiento los supuestos positivistas de la realidad objetiva que pueda ser explicada a través de la ley de la causalidad y las leyes universales, las cuales dilucidan las limitaciones de la investigación cuantitativa del pensamiento científico-naturalista de la investigación educativa. Ambos enfoques -tanto el positivista como el interpretativo- coinciden en que el investigador y el acto de investigar permanecen ajenos a la situación y toman una postura desinteresada que rechaza cualquier interés de las realidades educativas que se analizan y se desee cambiarlas. 


\section{La comunicación como eje central de la interpretación en la investigación educativa}

Este tema considerado un problema por las diferentes visiones y acepciones que el lector puede dar a "La sociología de la educación, del funcionalismo a la fenomenología", tendrá su enfoque en lo relacionado con los acontecimientos que dan origen a la sociología de la educación entendidos como historia que fundamenta esta ciencia.

Los actores sociales son quienes actúan en el proceso de cambio de una sociedad, afectando de manera positiva o no, las transformaciones que en ella se producen. Es en estos procesos en los que se promueven comportamientos culturales de orden social; el hombre desde sus diferentes dimensiones construye relaciones que dan sentido a la realidad objetiva social y cultural.

En este sentido para abordar desde la pedagogía la sociología de la educación, se presentará el punto de vista de su origen apoyado en los elementos propuestos, por la que se interpreta el sentido de ésta en la organización social, planteamiento que acorde con la investigación y a partir de varios aspectos, involucra el comportamiento del hombre. Por ejemplo: la práctica de la religión como fundamento del desarrollo moral, la educación como la diferenciación entre lo científico y lo escolar, dándole prioridad a su estudio a través de las concepciones que de manera particular se forma cada docente para que a su vez otros (estudiantes) entiendan la relación entre sociología y educación, además de conocer las interrelaciones de los actores de ésta.

En la Modernidad existe una serie de enfoques que, como se viene diciendo, comprometen una serie de interrelaciones, hecho por el cual se debe considerar su estudio comenzando por observar la cotidianidad de sus actores; pues en las comunicaciones sociales, la fenomenología participa de la construcción real de sociedad, 
permitiendo cuestionar lo que se da en la cotidianidad. Es así como de acuerdo con el enfoque que se le dé a la educación, se interpretan la organización, las lecturas que se hacen de lo cotidiano, el sistema de valores y de sociedad que se comparte.

En este orden de ideas, se plantea la dificultad que se tiene desde el positivismo para comprender la realidad social. Su descubrimiento parte de la pauta social que va marcando la cotidianidad proponiendo que el hombre no es el actor principal de construcción de la realidad, negando la intervención del sujeto por ser determinante; no admite del estudiante una mentalidad abierta, motivando únicamente a la descripción de hechos. Coarta las propuestas puesto que los hechos se ven de manera singular e individual y parecería que sin conexión entre uno y otro, de hecho Dilthey, afirma que las ciencias sociales tienen la capacidad de explicar los sucesos humanos aplicando métodos que le sean propios y que posteriormente ese entretejido deje de ser experimental y se interpreten sus significaciones.

De lo anterior se deduce que las ciencias sociales por ser subjetivas, $y$, al estudiar la cultura en el orden que la estructura, emergen las interpretaciones como resultado de las interacciones entre lo histórico y el significado que se le da a lo que se observa y que a la vez es construido por él mismo (el hombre). Es decir, le da significado a lo que ve, estas "consideraciones subjetivas" abren paso a las similitudes y a la multiplicidad como representaciones de la realidad.

Se comprende entonces, que la vida social gira en torno a la acción humana y a la dimensión de la subjetividad, como bien se deduce del postulado de Gadamer, en el que plantea que el objetivo de la hermenéutica integra la aplicación con la comprensión y la explicación como parte constitutiva de este proceso, que además es confirmado por Wittgenstein al describir la acción social como la integradora entre la filosofía del lenguaje que constituye la 
actividad misma; el pensamiento de la fenomenología social y la comprensión intersubjetiva.

Parece claro hasta ahora, que al relacionarse la sociología con la pedagogía, esta última pasa a integrar la comprensión de las interacciones sociales y sus actores, su contenido y forma de transmitirlo, son clave para comprender los componentes que pertenecen o son propias de una cultura, razón por la cual se considera que desde la pedagogía se da paso a la interpretación de los hechos sociológicos y culturales que en el aula se conocen y del que subyacen una organización para la transmisión de los mismos, dilucidando actitudes y acciones del hecho social; es entonces que el docente interviene con un papel fundamental frente a estas acciones dentro del aula, es decir, que desde la interpretación que realiza para dar a conocer el hecho, puede incidir como absolutista o como transformador, generando actitudes críticas, reflexivas y de compromiso con la misma sociedad, que piensen críticamente sobre los principios que estructuran la vida y la práctica en el aula.

La educación toma en cuenta la interacción social en donde el lenguaje juega un papel importante que da lugar a la intersubjetividad en lo social en el aula.

En este sentido se considera pertinente abordar el tema del conocimiento haciendo referencia a cómo la epistemología que da origen a la investigación hermenéutica es una propuesta de investigación de procesos cualitativos, que permiten interpretar el contenido social con las características mencionadas, es decir, motivar procesos de interpretación y comprensión de la acción humana que se constituye en el objeto de estudio en el contexto del que surgen. Los estudiantes desde su práctica formativa comienzan a establecer diferentes tipos de relaciones y de interacciones que les permiten inquietarse por investigar esos hechos de los que de alguna manera vienen a formar parte. Idea que se afirma con el 
pensamiento de Droysen al decir que: "el ser humano expresa su interioridad mediante manifestaciones sensibles y toda expresión humana sensible, refleja una interioridad. No captar, por tanto, en una manifestación, conducta, hecho histórico o social esa dimensión interna, equivale a no comprenderlo" (Arquitectura de la Historia, 1858).

En articulación con las anteriores ideas se retoma de nuevo a Gadamer, quien permite comprender qué parte del comportamiento humano es la palabra como hecho inherente a él. Gadamer asevera que la comprensión no sólo está en lo que reflejan sus actitudes y comportamientos, va más allá, es entender que las relaciones se dan a partir del lenguaje pues este es el medio por el que los participantes de una sociedad llegan a acuerdos.

Gadamer, (1993 p. 378) promotor del enfoque lingüístico o del lenguaje como experiencia hermenéutica, agrega que ésta tiene tres momentos: la subtilitas intelligendi o comprensión, las subtilitas explicandi o interpretación y las subtilitas applicandi o aplicación. Entendida subtilitas como un método disponible que como un saber hacer que requiere una particular -finura del espíritu-. En consecuencia se amplían las posibilidades de interpretaciones acentuando la subjetividad que constantemente habla la teoría interpretativa puesto que esta acción permite dar el punto de vista desde las propias creencias, la propia práctica de los valores aplicados por sus coetáneos alejando la posibilidad de aceptar otras teorías que pretendan explicar las vivencias, la cotidianidad del grupo social. De ello se infiere que ahí es donde radican y se acentúan las diferentes ideologías, esas ideologías propias de una realidad social defendidas y cimentadas por sus miembros.

En definitiva, el arte de la palabra se convierte en una herramienta fuerte en el momento de llegar a acuerdos y consensos para lograr modificar o por lo menos incidir en el pensamiento de otros ¿̇Cómo 
lograrlo?: obrando de manera correcta, con compromiso reflexivo y crítico fundamentando el punto de vista con argumentos convincentes fundada en esa ciencia, la ciencia social interpretativa.

El problema de interpretar es tener la certeza de que lo interpretado tendrá puntos de controversia y la poca posibilidad de lograr los cambios que la elucidación ha querido aclarar en "pro" de una comunidad.

\section{Conclusiones}

Resulta imprescindible que el investigador educativo sea capaz de conocer, identificar y manejar adecuadamente todas las técnicas y métodos del conjunto metodológico del cual se pueden servir para dar cumplimiento al objetivo que se ha trazado para su trabajo.

Para garantizar una preparación profunda de los investigadores educativos, es necesario que en su periodo de información deban enfrentar la realización de trabajos que incluyan las diferentes estrategias investigativas, lo cual les facilitará una preparación más general y los pondrá en condiciones de enfrentar con mayores posibilidades de éxito su labor profesional.

La relación entre teoría-práctica es una relación intensa, pues el conocimiento hermenéutico permite a los grupos sociales conocerse a sí mismos y a la realidad que los rodea, la cual está llena de intenciones e intereses de quienes participan.

Enseñar y aprender no se reduce a las relaciones profesor - estudiante, en este proceso intervienen: la cultura como experiencia vital del mundo fenomenológico, la interpretación social es una manera de construir la educación que logre trascender las paredes del aula con profesionales agentes de proyección social creadores de una verdadera transformación. 


\section{Bibliografía}

CARR, Wilfred y KEMMIS Stephen. Planteamiento Interpretativo de La Teoría Y Prácticas Educativas. Campo de Formación Investigativo. Página 31 a la 40

SANDIN, María Paz. (2003). Investigación Cualitativa en Educación. Capítulo 3. Perspectivas Teórico - epistemológicas en la investigación educativa. Madrid, McGraw-Hill, 2003

BERNABEU Rico y COLOM, Cañellas. El paradigma crítico hermenéutico y el paradigma tecnológico. Material de lectura para Epistemología de la ciencia. Recopilado Marín Gallego J. (2010).

GADAMER, Hans-George. (1993). Verdad y método. T.I. Salamanca, Sígueme

HERNÁNDEZ, Ana I. (2003). Revista internacional. Venezuela

HABERMAS, Jürgen. (1990). La Lógica de las ciencias sociales. Madrid, Ed. Tecnos.

INFORME MAESTRO III. (2010). Módulo Investigativo. Campo de Formación Investigativo. Universidad Santo Tomás. MARIN, Gallego José Duván. (2009). Procesos de Teorización y conceptualización. Universidad Santo Tomás.

Recibido en diciembre 2010

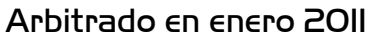

